\title{
Étalement urbain et différenciation sociospatiale à Dakar
} (Sénégal)

\author{
Urban sprawl and socio-spatial differentiation in Dakar \\ (Senegal)
}

\section{Expansión urbana y diferenciación socio-espacial en Dakar (Senegal)}

\section{Ibrahima Ndiaye}

Volume 59, numéro 166, avril 2015

Version originale soumise en juillet 2014. Version révisée reçue en avril 2015.

URI : https://id.erudit.org/iderudit/1034348ar

DOI : https://doi.org/10.7202/1034348ar

Aller au sommaire du numéro

Éditeur(s)

Département de géographie de l’Université Laval

ISSN

0007-9766 (imprimé)

1708-8968 (numérique)

Découvrir la revue

Citer cet article

Ndiaye, I. (2015). Étalement urbain et différenciation sociospatiale à Dakar (Sénégal). Cahiers de géographie du Québec, 59(166), 47-69.

https://doi.org/10.7202/1034348ar
Résumé de l'article

Les villes du Sud sont caractérisées par un étalement urbain, généralement accompagné d'une dynamique de différenciation sociospatiale très prononcée. Le contexte de la ville de Dakar, principal pôle attractif, concentrant les fonctions les plus importantes du territoire national sénégalais, est utilisé ici pour mettre en place une démarche méthodologique permettant d'analyser la différenciation sociospatiale. En convoquant les méthodes d'analyse multidimensionnelle et en nous basant sur les données issues d'enquêtes ménages originales, nous nous sommes donné l'objectif d'analyser la différenciation sociospatiale à Dakar. Il ressort de nos analyses que l'agglomération dakaroise est sujette à un étalement urbain, avec l'échec des politiques de planification du développement urbain et la déréglementation du marché du foncier et de l'immobilier, qui ont été sources d'exclusion des populations à faible revenu. L'analyse de ce territoire étalé montre un espace complexe, animé de dynamiques de différenciation sociospatiale très marquées. 


\title{
Étalement urbain et différenciation sociospatiale à Dakar (Sénégal)
}

\author{
Urban sprawl and socio-spatial \\ differentiation in Dakar (Senegal) \\ Expansión urbana y diferenciación socio- \\ espacial en Dakar (Senegal)
}

\author{
Ibrahima NDIAYE \\ École Supérieure d'Économie Appliquée, \\ Université Cheikh Anta Diop , Dakar \\ Ibrahima.Ndiaye@univ-mlv.fr
}

\section{Résumé}

Les villes du Sud sont caractérisées par un étalement urbain, généralement accompagné d'une dynamique de différenciation sociospatiale très prononcée. Le contexte de la ville de Dakar, principal pôle attractif, concentrant les fonctions les plus importantes du territoire national sénégalais, est utilisé ici pour mettre en place une démarche méthodologique permettant d'analyser la différenciation sociospatiale. En convoquant les méthodes d'analyse multidimensionnelle et en nous basant sur les données issues d'enquêtes ménages originales, nous nous sommes donné l'objectif d'analyser la différenciation sociospatiale à Dakar. Il ressort de nos analyses que l'agglomération dakaroise est sujette à un étalement urbain, avec l'échec des politiques de planification du développement urbain et la déréglementation du marché du foncier et de l'immobilier, qui ont été sources d'exclusion des populations à faible revenu. L'analyse de ce territoire étalé montre un espace complexe, animé de dynamiques de différenciation sociospatiale très marquées.

\section{Mots-clés}

Différenciation sociospatiale, ségrégation, étalement urbain, analyse multivariée, enquêtes de terrain, Dakar.

\begin{abstract}
One characteristic of southern cities is urban sprawl, usually in combination with a highly marked dynamic of socio-spatial differentiation. The situation in the city of Dakar, Senegal's main pole of attraction where the most important functions of the nation's activities are carried out, was used to develop a methodological approach for an analysis of socio-spatial differentiation. The purpose was to draw on multidimensional analysis methods and actual household survey data to analyze socio-spatial differentiation in the city. Our study shows that the Dakar area is vulnerable to urban sprawl as a result of the failure of the city's urban development planning policies, and deregulation of the land and housing markets, factors which have become sources of exclusion for low-income groups in the population. Further analysis of this sprawling urban area reveals a complex space, governed by a strongly marked dynamic of socio-spatial differentiation.
\end{abstract}

\section{Keywords}

Socio-spatial differentiation, segregation, urban sprawl, multivariate analysis, field studies, Dakar. 


\section{Resumen}

Las ciudades del Sur se caracterizan por una expansión urbana generalmente acompañada de una dinámica de diferenciación socio-espacial muy marcada. El contexto de la ciudad de Dakar, principal polo de atracción, que concentra las funciones más importantes del territorio nacional senegalés, sirve aquí para aplicar un proceso metodológico que permita analizar la diferenciación socio-espacial. Utilizando métodos de análisis multidimensional y datos domésticos originales, nuestro objetivo es el análisis de la diferenciación socio-espacial en Dakar. Resulta, de nuestros análisis, que la aglomeración de Dakar está sometida a la expansión urbana, con el fracaso de las políticas de planificación de desarrollo urbano y de la liberalización del mercado de terrenos y bienes inmuebles, siendo fuentes de exclusión de la población con menguado ingreso. El territorio expandido muestra un espacio complejo, animado por las dinámicas de diferenciación socio-espacial bien marcadas.

\section{Palabras claves}

Diferenciación socio-espacial, segregación, expansión urbana, análisis multivariado, encuestas de terreno, Dakar.

\section{Introduction}

Dakar est une agglomération cosmopolite, où l'on note une grande diversité du tissu urbain, avec la cohabitation entre zone lotie et non lotie, l'habitat régulier et irrégulier, l'habitat individuel et collectif, etc. Cette diversité témoigne d'une certaine hétérogénéité sociale, avec une cohabitation entre les riches et les pauvres, les secteurs formel et informel, etc. Il faut dire que le dualisme, qui veut que les zones centrales et périphériques soient respectivement peuplées par des populations riches et pauvres, n'est pas toujours établi. Nous pouvons donner l'exemple du quartier irrégulier de Rebeuss, jadis zone tampon séparant le centre-ville de la Médina (quartier des indigènes) et qui, aujourd'hui, est un bidonville situé dans les interstices de l'hyper-centre.

Une démarche facile serait donc de considérer que les populations pauvres sont localisées dans la périphérie et que le centre concentre l'habitat moyen et riche. Ce modèle est inopérant dans la capitale sénégalaise. En effet, comme le disent Diaz Olvera et Godard (2002), l'urbanisation périphérique mal contrôlée renforce les difficultés pour les nouvelles populations localisées à la frange urbaine, qui sont souvent parmi les plus pauvres, mais non exclusivement, car on y trouve également des ménages aisés.

Pour montrer toute l'hétérogénéité dans l'occupation de l'espace urbain, nous proposons, dans le cadre de cet article, de mettre en lumière les dynamiques de différenciation sociospatiale en œuvre à l'échelle de l'agglomération dakaroise. Nous ne nous inscrivons pas dans une analyse des mécanismes ayant abouti à une différenciation sociospatiale ${ }^{1}$. Plusieurs questions émergent: Quelle démarche

1 Dans le cadre de ce travail, nous préférons utiliser le terme «différenciation sociospatiale», lequel nous semble plus neutre que «ségrégation», qui est le fruit d'un processus de lutte de pouvoir et de rapport de force. Nous pensons qu'il n'existe plus aujourd'hui à Dakar de ségrégation, entendue comme une politique de mise à l'écart et de séparation des classes sociales. 
méthodologique pour mettre en exergue la différenciation sociospatiale? Quelles sont les données disponibles et leurs limites? À quelle échelle traiter les phénomènes de différenciation sociale de l'espace?

\section{Contexte}

L'étalement urbain (urban sprawl) traduit l'extension de la superficie d'une agglomération sur le territoire avoisinant. Cependant, ce concept est plus précis que l'extension, qui désigne l'action de développer, d'accroître les dimensions. L'étalement fait référence à une diminution dans l'intensité de l'occupation du sol urbain (Pouyanne, 2004). C'est «une croissance discontinue et à faible densité des villes» (Ascher, 2004: 29).

Ce processus d'étalement urbain est rendu possible par les nouvelles techniques de communication, qui ont permis non seulement de gagner du temps, mais surtout d'augmenter la portée spatiale des déplacements en maintenant relativement stable le budget temps de transport de chaque individu (Kaufmann, 2003). Selon la conjecture de Zahavi, le gain de temps n'est pas généralement utilisé pour faire d'autres activités, mais est réinvesti dans de la distance supplémentaire, pour élargir la portée spatiale des déplacements de la vie quotidienne, permettant ainsi aux ménages d'aller habiter plus loin de leur lieu de travail. D’autres facteurs contribuent aussi à l'accélération de ce processus, qui a abouti à un archipel urbain : le mythe de la propriété individuelle, les prix fonciers bas ou la proximité de la nature en périphérie.

La forme urbaine a pendant longtemps été une source de controverse entre les différents chercheurs. Pour les tenants de la ville étalée, l'augmentation des distances à parcourir est un faible coût comparé aux avantages de ce type de ville, qui permet une occupation plus uniforme du territoire, mais aussi un accès plus facile à la propriété foncière pour les populations. Pour ces partisans de la diffusion urbaine, Gordon et Richardson cités par Massot et Orfeuil (2004), la ville étalée est synonyme d'efficacité économique et sociale. Les tenants de cette approche font l'impasse sur les problèmes environnementaux que soulève l'étalement urbain. La deuxième approche, qui fait aujourd'hui de plus en plus autorité, plaide pour une ville compacte avec une mixité sociale et fonctionnelle. Cependant, cette posture «fait l'impasse, dans l'absolu, sur la prégnance, dans les zones denses et au moins dans l'imaginaire collectif, des inconvénients de la densité, et aussi sur des difficultés d'accès au logement» (Massot et Orfeuil, 2004: 225). Pour Wiel (2010), cette approche est utopique et sa mise en œuvre renforcera les inégalités et sera source de discrimination dont souffriront les plus démunis. Wiel plaide plus pour une maîtrise de la croissance urbaine et le renforcement de l'attractivité des moyens de transport collectif.

La pression foncière, dont les villes font l'objet, constitue un facteur d'exclusion des plus pauvres. L'augmentation des prix du foncier et de l'immobilier est à l'origine d'une différenciation spatiale qui se manifeste dans les villes du Sud, comme Dakar, par une périphérisation des couches moins aisées et l'accaparement du centre, proche des lieux de décision, par les couches nanties. Avec l'étalement urbain, le transport occupe une part de plus en plus importante dans le budget des ménages périurbains modestes. Il constitue le troisième poste de dépense pour les ménages dakarois, après les dépenses alimentaires (Agence nationale de la statistique et de la démographie, 
2005). La cherté du coût du transport ${ }^{2}$ maintient ces populations dans leur situation d'«insularité» et les pousse à se cantonner dans des espaces restreints avec peu de moyens pour accéder aux commodités et aux ressources urbaines.

Cette périphérisation des couches moins aisées, ainsi cantonnées dans les zones lointaines où les prix du foncier sont très bas, est assimilable à une différenciation sociospatiale, qui est une forme plus ou moins institutionnalisée de la distance sociale, traduisant une séparation dans l'espace (Fijalkow, 2007). Cette quête d'une ville homogène n'est pas un phénomène nouveau. Déjà au XIXe siècle, les classes dominantes, souhaitant des villes socialement homogènes, reléguaient les classes laborieuses, jugées dangereuses et porteuses de maladies, dans des espaces périphériques.

Dans la tyrannie des petites décisions, Schelling, cité par Grafmeyer et Authier (2008), propose de distinguer trois processus essentiels de la différenciation spatiale: le premier type de processus découle d'une action volontaire et réfléchie visant implicitement ou explicitement la séparation physique entre diverses catégories de populations. La création de la Médina, à Dakar, est un exemple ${ }^{3}$. Le second est plus lié aux inégalités produites par la différenciation sociale, indépendamment de toute intention. Lié à la situation économique des ménages, ce processus pourrait aboutir à une séparation entre les pauvres et les riches, les moins instruits et les plus instruits, etc. Le troisième processus concerne les acteurs, dont le jeu combiné des critères de choix résidentiels aboutit à des situations de différenciation sociale sans que cela soit un objectif. Cette séparation entre les catégories de populations se traduit par des spécificités dans l'occupation de l'espace urbain. Les coûts du transport, l'accès aux services sociaux de base, la proximité du centre sont autant de déterminants des prix des terrains en zone urbaine.

Ce travail de recherche, s'intéressant à la différenciation sociospatiale dans les espaces urbains, se positionne aussi par rapport à ceux menés sur Dakar depuis plusieurs années et qui cherchaient à mettre en exergue l'hétérogénéité de cet espace (Seck, 1970; Vernière, 1973 et 1977 ; Salem, 1998; Lombard et al., 2006 ; Lessault et Sakho, 2008 ; Lessault et Imbert, 2013). Déjà dans les années 1970, Marc Vernière notait l'existence de cette dynamique ségrégative: «[...] à Dakar, au sein des différents quartiers, la ségrégation sociale est si forte, la spécialisation si poussée [...]» (Vernière, 1977: 17). Les effets cumulés de l'échec des politiques d'habitat planifié et de maîtrise foncière ont renforcé cette dynamique de différenciation sociospatiale. En effet, avec le renchérissement des tarifs, l'accès à la propriété immobilière et foncière devenait presque impossible pour les catégories sociales pauvres (Mbow, 1992). Dans ce contexte, les Lébous tolèrent, moyennant finance, l'installation des citadins rejetés par la grande ville (Vernière, 1973).

Ainsi, on assistait à un développement urbain en mosaïque marqué par une imbrication de quartiers d'habitat planifié aménagés en centre-ville par les sociétés immobilières publiques (Seck, 1970), de quartiers populaires en zone péricentrale (Vernière, 1973 ; Mbow, 1992) et de quartiers naissants (Pikine-Dagoudane) devant accueillir les néo-

2 Prétextant une hausse des prix du carburant, les propriétaires des minibus TATA ont augmenté unilatéralement, le 16 décembre 2011, les tarifs de 15 à $30 \%$.

3 Le lotissement de la Médina, qui date de 1914, a mis en place pour séparer les colons des populations indigènes. L'épidémie de peste a servi de prétexte pour fonder la décision de séparation des classes. 
urbains venus du monde rural en banlieue (Salem, 1998). On note aussi la présence éparse de zones d'habitat traditionnel (villages traditionnels) situées sur le littoral dakarois (Seck, 1970) et, plus récemment, de quartiers résidentiels marqués par les migrations internationales (Tall, 2009). Cet espace urbain évolue et se différencie: pendant que certains lieux se trouvent valorisés ou renforcés, d'autres passent en retrait ou sont amoindris (Lombard et al., 2006).

\section{Fondation et organisation du développement urbain de Dakar}

Dakar a toujours joué un rôle économique et politique important. La ville a traversé différentes phases dans son développement: d'abord cité symbole de la traite négrière avec l'île de Gorée, ensuite pôle de colonisation, en étant la capitale de l'Afrique occidentale française (AOF), et aujourd'hui première ville par sa taille devant Touba.

Les colons se sont d'abord installés à Gorée et ne voulaient pas cohabiter avec la population indigène. Les autorités coloniales craignaient de s'installer sur la terre sénégalaise, qui était une "terre de fièvre et de barbarie» (Sinou, 1985). La démographie galopante sur la petite île de Gorée, qui devenait de plus en plus étroite, a poussé le capitaine de vaisseau Protet à fonder la ville, en 1857. PétuaudLétang (2010) définit deux modalités de création d'une ville. La création peut être spontanée: sur un site particulier, un peuple nomade décide de se fixer, le lieu étant propice au développement d'activités lucratives, dans un environnement apte à nourrir les gens en permanence. C'est dans un tel contexte que les Lébous se sont installés dans la région de Dakar. La création de la ville peut aussi découler d'une décision politique qui l'impose. La forme préexiste dans le choix du décideur et un plan rationnel, théorique, est imposé à l'espace et aux gens. Cette deuxième modalité est caractéristique de la fondation de la ville de Dakar par le colonisateur.

La gestion foncière a toujours été un épineux problème pour le développement de la région de Dakar. La non-reconnaissance formelle des droits coutumiers lébous par le colonisateur a amené les occupants à mettre en place un circuit de gestion parallèle, qui a participé au développement des quartiers spontanés. Tous ceux qui étaient exclus du système formel se sont rabattus sur les Lébous pour accéder à la propriété foncière. Les Lébous concédaient les parcelles à «certains déguerpis de Dakar. Pikine irrégulier s'est donc constitué à partir de la ville de Pikine à proprement parler et des villages lébous de Thiaroye gare, Thiaroye Kao et Yeumbel. Ces différents pôles se sont rejoints et ont finalement formé cette zone urbaine» (Sy, 1991 : 106).

Pour occuper les sites stratégiques, le colonisateur a mis en place une stratégie d'accaparement qui a été légitimée par les règles d'urbanisme et d'hygiène. Ces différents outils sont marqués par le sceau de la ségrégation.

La coexistence entre les deux communautés (française et autochtone), aux cultures spécifiques et intérêts divergents, n'est pas sans créer des heurts, surtout en matière de foncier. Devant leur volonté de s'accaparer du Plateau, qui est la zone stratégique, les colons mûrirent l'idée de déplacer les indigènes en dehors des limites du Plateau. Pour les autorités coloniales, la seule manière d'assurer leurs tâches de colonisation était précisément la séparation des communautés (Seck, 1970). 
Pour atteindre cet objectif, une première stratégie mise en œuvre était d'édicter des règles coercitives d'hygiène et d'urbanisme. En 1905, le lieutenant-gouverneur du Sénégal institua l'obligation d'une autorisation pour construire à Dakar et interdit l'utilisation de matériaux légers tels que banco, paillotes, cartons, etc. Il est clair que cette mesure visait principalement les habitations indigènes, dont la plupart étaient construites avec des matériaux précaires. Cette stratégie était peu efficace. Certains indigènes, disposant de ressources financières, parvenaient à respecter ces règles et donc à rester dans la zone du Plateau.

La deuxième stratégie, plus radicale, était de créer une «ville indigène ». L'épidémie de peste qui s'est déclarée en 1914 va servir de prétexte au dessein colonial. L'arrêté no 1467 du 19 septembre 1914, signé par le gouverneur William Ponty, concrétise le projet. Une bonne partie de la population autochtone est déplacée vers Tiléne, une vaste plaine marécageuse située au nord du Plateau. Occupé par les champs, le site est d'abord asséché puis loti selon un plan en damier (Sakho, 1985 ; Ndiaye, 2009).

La fondation et le développement de Dakar ont été marqués par une ségrégation spatiale, qui visait à séparer les deux classes: indigène et européenne. Cette dualité a affecté l'organisation de la ville, avec une urbanisation à deux vitesses. Une ville coloniale bien organisée, avec des règles d'urbanisme strictes et une ville indigène désarticulée, principalement construite en baraques. La différenciation spatiale caractérisant la ville se traduisait aussi dans le domaine du foncier. Dans les quartiers européens, le droit de propriété, qui procurait une certaine stabilité et sécurité, était octroyé, alors que dans les zones occupées par les indigènes, les permis d'habiter et d'occuper étaient attribués à titre précaire et révocable. C'est dans ce contexte que les premiers outils de planification ont été élaborés pour organiser le développement urbain.

\section{Dynamique urbaine et étalement à Dakar}

Bien que plusieurs documents de planification aient été produits, il faut reconnaître qu'ils ont toujours été en retard par rapport aux réalités sur le terrain. Ces documents ont été vite dépassés parce que n’ayant jamais réussi à prévoir le croît démographique important que la ville a connu. Les populations, non impliquées lors de la conception de ces outils de planification, n'en ont de plus jamais compris la pertinence et l'importance. Lors de la colonisation, ces documents ont toujours été perçus comme un outil du colonisateur pour exclure les indigènes de la ville. Cette perception est restée ancrée chez les gens bien après la colonisation. Les règles de zonage n’ont jamais été traduites sur le terrain, ce qui a fait que certaines zones non aedificandi ont été occupées au mépris des règles de servitude. L'insuffisance des ressources permettant de mettre en œuvre les projets prévus, le non-respect des règles de servitude et de construction ainsi que la non-implication des populations, qui ont toujours perçu l'urbanisme comme un moyen de domination et d'exclusion ne respectant pas leurs us et coutumes, ont été à l'origine d’un développement spatial désordonné et extensif.

L'occupation de la presqu'île par Pinet Laprade a amorcé le processus d'urbanisation. La zone urbanisée était contiguë au port de Dakar et s'étendait sur environ $1 \mathrm{~km}^{2}$. À cette époque, la mixité sociale était présente avec la cohabitation des colons et des 
populations autochtones. De faible extension territoriale, cette ville piétonne avait la marche comme principal mode de déplacement. L'absence de moyens de transport motorisés déterminait ce type de configuration spatiale compacte. À côté de la zone urbanisée, subsistaient des villages traditionnels lébous situés sur la frange maritime. La création de la Médina, en 1914, a été la première extension de la ville. Les populations autochtones ont été déportées vers le nouveau lotissement, séparé du quartier européen par un cordon sanitaire, et devenu aujourd'hui le quartier de Rebeuss.

Avec l'extension de la ville, les problèmes de transport urbain ont commencé à se manifester. L'allongement des distances à parcourir et l'utilisation de la marche, comme principal moyen de transport rendaient les déplacements difficiles. Ainsi, en 1932, fut mis en place le premier réseau de transport urbain. Cette première expérience, qui provenait du secteur privé, voyait la création de la Compagnie sénégalaise de transport en commun (CSTC). Cette compagnie ne couvrait que les quartiers du Plateau et de la Médina. Les villages traditionnels, avec l'absence de réseau viaire étaient exclus de cette desserte de transport.

La création de la Société immobilière du Cap-Vert (SICAP) ainsi que de la Société nationale des habitations à loyer modéré (SNHLM) ont accéléré le processus d'étalement avec la mise en œuvre de nouveaux lotissements destinés à la classe moyenne. En 1955, la superficie urbanisée était d'environ $30 \mathrm{~km}^{2}$ pour une population estimée à 214000 habitants. À la suite de la création de Pikine, Dakar devient bicéphale: le centre-ville et ses extensions, et la zone périphérique avec les occupations irrégulières.

Dakar et Pikine forment aujourd'hui une conurbation très densément occupée, découlant des lotissements effectués par les promoteurs immobiliers privés et publics (SICAP et SNHLM), surtout avec le projet des parcelles assainies, qui s'étendait sur plus de 400 ha. L'habitat dans ce continuum urbain (figure 1) est de type horizontal, avec très peu d'immeubles de très grande hauteur. Les seuls espaces inoccupés sont l'aéroport de Dakar ${ }^{4}$, la zone des Niayes et la forêt classée de $\mathrm{Mbao}^{5}$. Le développement se fait par l'occupation de certains espaces situés dans les interstices du tissu urbain, avec un changement de vocation ${ }^{6}$, dans les espaces du domaine public maritime et en périphérie de l’agglomération.

Les communes d'arrondissement sont presque totalement occupées. Seules celles de Keur Massar et de Malika disposent encore de réserves foncières habitables. L'extension future de la ville va se faire sur l'espace rural, créant du coup des conflits fonciers, ce qui posera un problème d'arbitrage entre les différentes fonctions. Le Plan directeur d'urbanisme de Dakar horizon 2025 privilégie l'habitat sur les autres fonctions économiques; ce faisant, toute la zone rurale sera urbanisée à l'horizon du Plan.

4 Situé à l'ouest, il sera fermé avec l'ouverture de l'aéroport international Blaise-Diagne, de Diass, en construction au moment de rédiger cet article. Les autorités prévoient en faire une zone d'affaires.

5 Située à l'est, elle est convoitée par les promoteurs immobiliers, qui souhaiteraient y voir des lotissements à usage d'habitation.

6 Le Centre international pour le commerce extérieur du Sénégal (CICES) et la bande verte, considérés comme des zones inconstructibles selon le Plan directeur d'urbanisme de Dakar, ont été lotis et les parcelles affectées à de tierces personnes, qui y ont construit des maisons à usage d'habitation. 


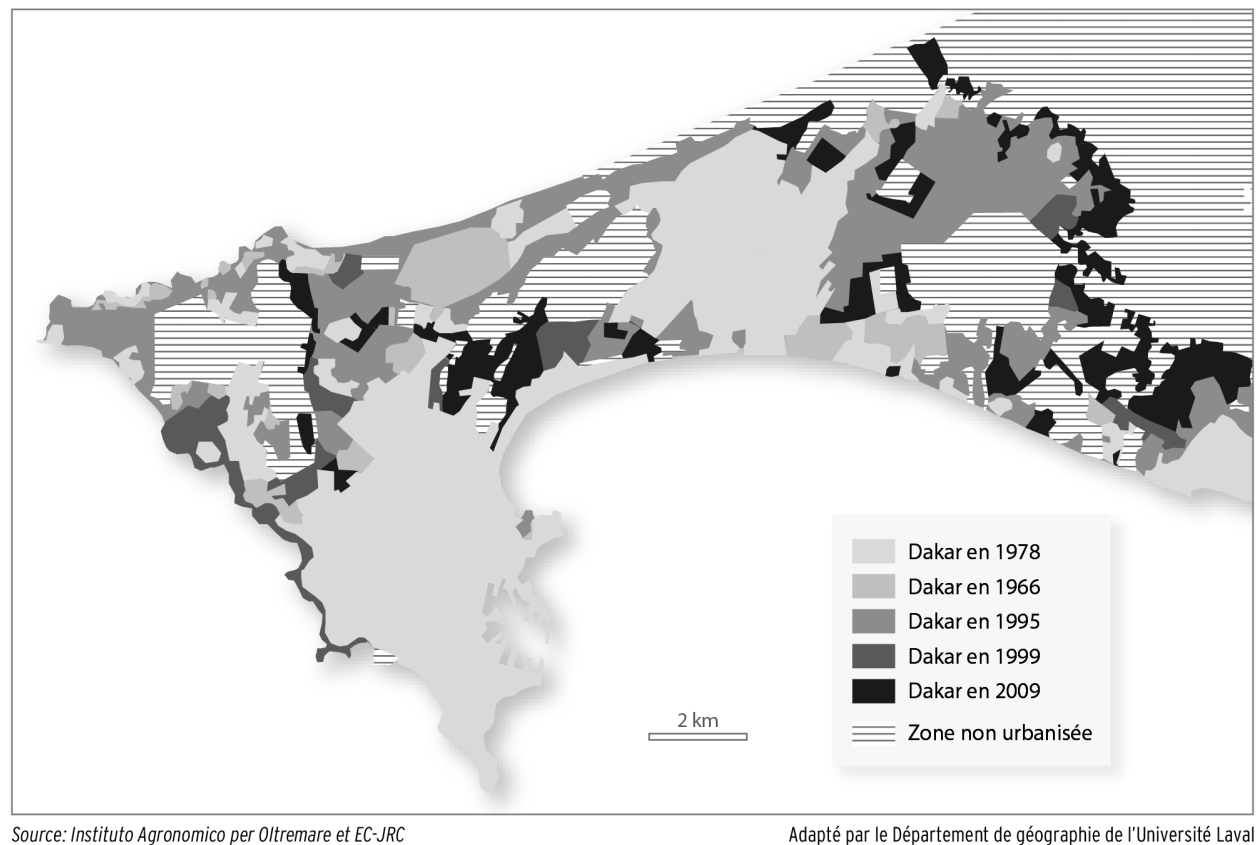
Conception: Ibrahima Ndiaye, Lab'Urba, 2011

L'étalement urbain est synonyme de dé-densification, la densité variant en fonction de l'éloignement ou de la proximité du centre-ville. La périurbanisation apparaît ainsi comme une discontinuité territoriale avec une faible densité et une faible diversité. Ce modèle explicatif n'intègre pas le cas de la région de Dakar, où les densités sont plus fonction du type d'habitat (figure 2). Dans les zones résidentielles, les coefficients d'emprise au sol (CES) et d'occupation du sol (COS) sont généralement très faibles alors que les parcelles y sont de grande taille ${ }^{7}$. Ce faible niveau d'occupation affecte la densité de population. Or, dans les zones d'habitat spontané, caractérisées par une promiscuité dans les chambres et un non-respect des règles d'urbanisme, le niveau d'occupation des parcelles est beaucoup plus important ${ }^{8}$. En effet, l'inadéquation, aussi bien quantitative que qualitative, entre l'offre de logement et la demande toujours croissante, contraint les ménages à des cohabitations «forcées», qui augmentent les taux d'occupation des logements (Lessault et al., 2011).

Les zones irrégulières de la banlieue ainsi que les zones populaires ${ }^{9}$, situées à proximité du centre-ville, sont les plus densément occupées. Les zones résidentielles ont les densités les plus faibles. Le front urbain à l'est de la région est en train de s'urbaniser,

7 Au Plateau, la superficie minimale d'une parcelle est de $400 \mathrm{~m}^{2}$ et, pour la zone des Almadies $500 \mathrm{~m}^{2}$.

8 Le coefficient d'emprise au sol atteint presque 100\% pour certaines maisons de la zone de GrandDakar. Les propriétaires, qui ne sont motivés que par l'argent, exploitent le moindre espace pour y construire une chambre à louer. Dans ces maisons, la consommation d'énergie est très élevée, du fait que, même en plein jour, on doit allumer la lumière pour voir.

9 Les quartiers Grand-Dakar, Médina, Grand-Yoff, les parcelles assainies et les villages traditionnels lébous. 


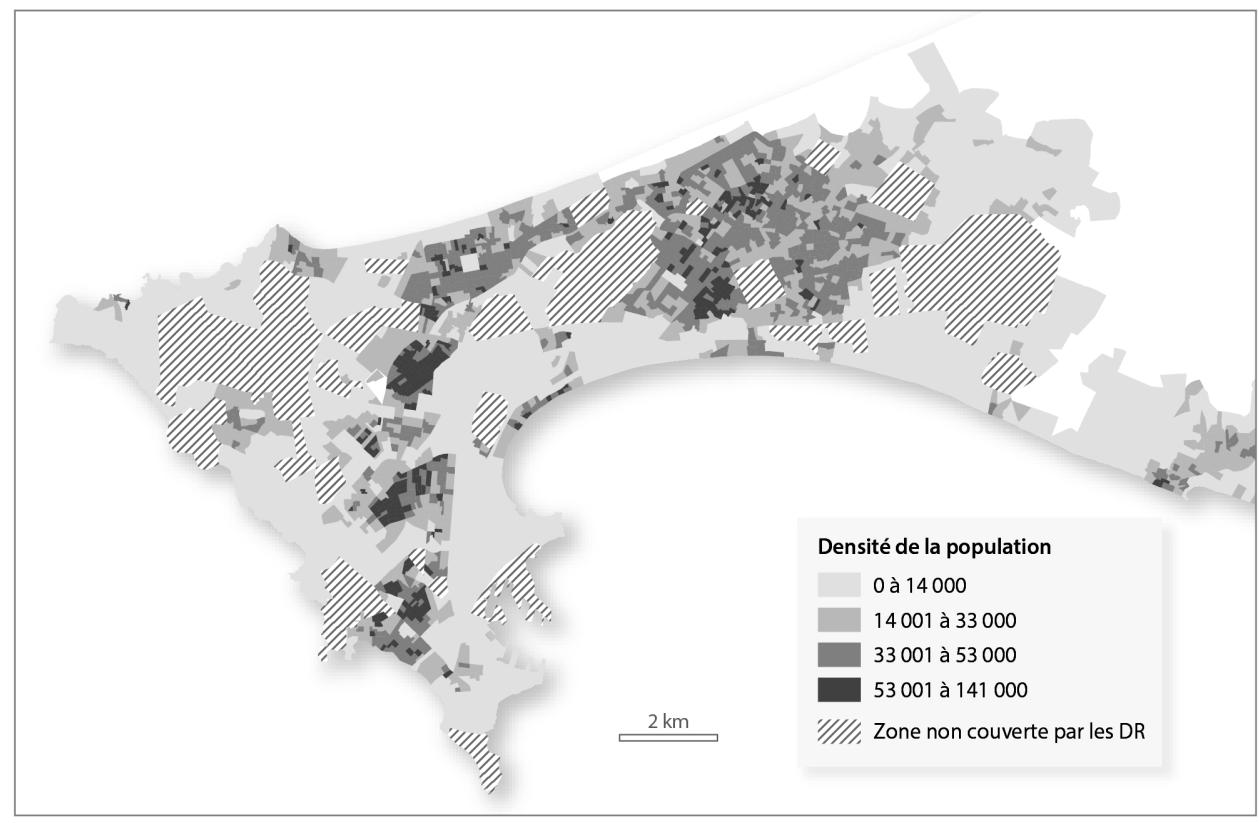

mais les densités y sont faibles, avec un habitat de type pavillonnaire et un faible niveau de construction des parcelles, issues des lotissements privés ou administratifs. Dans ce contexte, quels sont les facteurs explicatifs de cet étalement urbain?

\section{Déterminants de l'étalement urbain}

L'étalement urbain est un héritage des politiques publiques mises en œuvre depuis la période coloniale. Depuis la fondation de Dakar, les différentes politiques publiques ont cherché à repousser les populations pauvres, aux comportements et valeurs «non urbaines », vers la périphérie. L’objectif visé était de faire de Dakar une «ville» au sens plein du terme. Une ville se distinguant du monde rural par ses artifices et sa modernité. Attirés par les mirages de la ville, les ruraux sont venus s'installer à Dakar pour profiter des commodités. Ils ont toujours été rejetés vers la périphérie. Ce processus a été entamé en 1914 avec la création du quartier indigène de la Médina. Les autorités sénégalaises, bien après les indépendances, ont poursuivi la même stratégie pour «assainir» la ville. Cette politique a abouti au bicéphalisme de la région avec Dakar-ville, d'un coté, et Pikine-banlieue, de l'autre. Ces deux établissements, avec leur croissance démographique et spatiale, ont fini par former une conurbation dense, occupant toute la superficie urbanisée. L'étalement de la région est donc consubstantiel aux politiques publiques qui, en rejetant les populations pauvres vers la périphérie, ont privilégié la diffusion de la ville.

Cependant, à côté des politiques publiques organisatrices du devenir urbain, se développait dans la périphérie un marché foncier spéculatif organisé par les autochtones lébous. En effet, la non-reconnaissance formelle de leurs droits coutumiers par le colonisateur a amené les Lébous à mettre en place un circuit de gestion parallèle, 
qui a participé au développement de quartiers spontanés (Vernière, 1973). Tous ceux qui étaient exclus du système formel, se sont rabattus sur les Lébous pour accéder à la propriété foncière. Les Lébous concédaient, moyennant finance, les parcelles à certains déguerpis de Dakar. Ce marché parallèle, œuvre des populations autochtones, est aussi un facteur déterminant dans l'étalement de l'espace urbain dakarois.

La réglementation urbaine a participé à l'extension de la ville en ne privilégiant pas la densification des habitations. La hauteur des constructions, jusqu'à récemment, était limitée au rez-de-chaussée plus un étage $(\mathrm{R}+1)$ pour tous les lotissements de la SICAP et de la SNHLM. Les urbanistes, considérant la vocation résidentielle de cette zone, ne souhaitaient pas y promouvoir l'habitat en hauteur. Or, aujourd'hui avec la croissance démographique importante et l'amenuisement des terrains à lotir, la densification se présente comme un moyen efficace pour lutter contre les effets "pervers» de la spéculation foncière et locative. Prenant conscience de cela, les autorités ont changé la réglementation dans certaines zones, en 2008, notamment les SICAP, le Point E et le Plateau, pour permettre de résorber le déficit d'espace ${ }^{10}$. La hauteur des constructions est maintenant fonction de la largeur de la voie de desserte ${ }^{11}$ dans les lotissements de la SICAP (sauf pour les cités Mermoz et Sacré-Cœur). Sur le Plateau, qui est une zone mixte, aucune partie «n'est affectée d'un plafond de hauteur en termes absolus » ${ }^{12}$. Les normes de densité, ainsi que les coefficients d'occupation du sol (COS) et d'emprise au sol (CES) ont été intégrés dans le nouveau règlement. Cette verticalisation de l'habitat a des exigences en termes de calibrage des réseaux et de satisfaction de la demande sociale.

Le processus de la périurbanisation ne suit pas la même logique dans les pays du Nord et du Sud. Dans les pays développés, cette dé-densification est favorisée par l'aménagement de voies de circulation de très grande capacité, permettant de réduire les temps de parcours et le désir des classes économiques aisées d'avoir plus d'espace et un environnement moins pollué. Le coût élevé du foncier dans les centres-villes des pays du Sud pousse les gens de catégories moins aisées à aller habiter en périphérie, où la terre coûte moins cher et où le locatif est plus abordable. Les populations nanties préfèrent toujours rester à proximité du centre pour être proches des services et des aménités urbaines.

L'augmentation des vitesses, permettant d'accéder à des réalités urbaines distantes, pourrait accélérer ce processus de périphérisation des couches aisées dans les pays du Sud. Cependant, la congestion notée sur les différents axes desservant la banlieue dakaroise, ainsi que la faiblesse de la vitesse commerciale dans les transports collectifs ${ }^{13}$, n'incitent pas à la délocalisation en périphérie. Toutefois, avec les facilités d'accès qu'offrent les minibus TATA, la desserte est meilleure pour les populations habitant les zones périphériques. Dès lors, il apparaît que les logiques sont différentes: dans les pays du Nord, la diffusion est plus liée à l'augmentation de

10 Dans le rapport de présentation du décret $n^{\circ} 2008-913$ du 8 août 2008, le législateur met en avant l'urbanisation galopante de l'agglomération dakaroise, la pression foncière, le déficit d'espace et la forte demande de logement pour justifier la nécessité de revoir le règlement d'urbanisme dans les secteurs de Dakar Plateau et du Point.

11 La formule appliquée est $\mathrm{H}=1,3 \mathrm{~L}$ avec $\mathrm{H}$, la hauteur de la construction, et L, la largeur de la voie.

12 Décret $n^{\circ}$ 2008-913 op.cit.

13 La vitesse commerciale des bus de la société publique Dakar Dem Dikk sur les lignes de banlieue est d'environ $15 \mathrm{~km} / \mathrm{h}$. 
la vitesse permettant aux ménages de garder leur budget temps de transport constant et d'aller habiter plus loin de leur lieu de travail, alors que ce processus, à Dakar, est plus celui des populations pauvres à la recherche d'une propriété foncière.

Le processus d'étalement spatial analysé, nous passons à la présentation de notre démarche méthodologique permettant de mettre en exergue les dynamiques de différenciation sociospatiale en cours à l'échelle de notre espace d'étude.

\section{Méthodes d'analyse de la différenciation sociospatiale}

\section{Choix de l'échelle et des variables}

L’agglomération dakaroise, avec une population estimée en 2002 à environ 2,5 millions d'habitants, est constituée de quatre communes (Dakar, Pikine, Guédiawaye et Rufisque) réparties dans 1998 districts de recensement (DR) ${ }^{14}$. Certains districts ne sont pas couverts, soit du fait de leur caractère particulier (zones industrielles ou militaires), soit parce qu'ils ne sont pas habités (forêt classée, aéroports, zones dunaires, etc.). Notre zone d'étude se limite au découpage en DR effectué par l'Agence nationale de la statistique et de la démographie (ANSD) (figure 3).

\section{Figure 3 Découpage de la zone d'étude en districts de recensement}

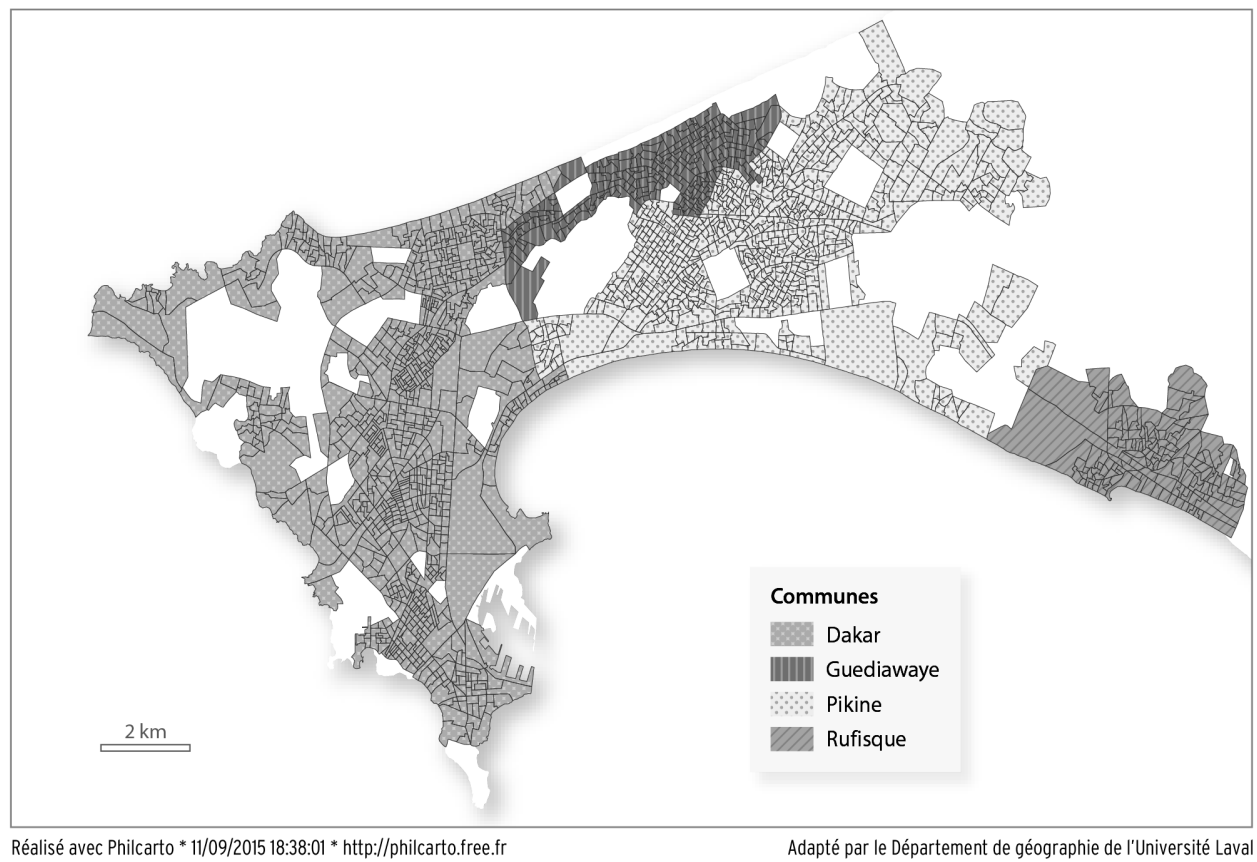

Pour faire ce travail de typologie spatiale, nous avons opté pour le découpage fin en districts de recensement, qui sert de base à l'ANSD lors du recensement général de la population. Ce découpage est le seul disponible sur Dakar avec des données aussi fines. Bien que les données soient anciennes, nous pensons que l'occupation de

14 Les districts de recensement comprennent en moyenne 1000 habitants pour environ 140 ménages. 
l'espace régional n'a pas évolué d'une manière très sensible entre 2002 et aujourd'hui. La zone urbaine, très densément occupée et la quasi-inexistence de réserve foncière, font que l'occupation du sol n’a pas évolué considérablement.

La base de données que nous avons obtenue à l'ANSD comportait 97 variables réparties en 14 groupes ${ }^{15}$. Du fait de la non-pertinence de certaines variables pour notre travail, notre choix s'est porté sur cinq groupes de variables ${ }^{16}$ (type d'activités, niveau d'équipements, matériaux de construction des logements, niveau d'instruction et type d'assainissement) comprenant au total 28 variables (tableau 1). Ces variables sont disponibles à l'échelle de tous les districts de recensement, ce qui permettait de faire un traitement statistique exhaustif à l'échelle de la ville.

Tableau 1 Liste des groupes et des variables retenus

\begin{tabular}{lll}
\hline \multicolumn{1}{c}{ Groupe de variables } & \multicolumn{2}{c}{ Variables } \\
\hline Assainissement & $\begin{array}{l}\text { Collecte porte à porte } \\
\text { Collecte charrette }\end{array}$ & $\begin{array}{l}\text { Égout } \\
\text { Latrine }\end{array}$ \\
& Dépôt sauvage & Fosse septique \\
& Toit en ciment & Sol ciment \\
Togement en banco & Sol carrelé \\
& Toit en bois & Sol en sable \\
& Télévision & Téléphone \\
Équipement & Vidéo & Cuisinière \\
& Réfrigérateur & Climatiseur \\
& Aucune instruction & Niveau secondaire \\
Instruction & Niveau primaire & Niveau supérieur \\
& Niveau moyen & \\
Activité & Actif occupé & Femme au foyer \\
& Chômeur & Retraité \\
& Élève/étudiant & \\
\hline
\end{tabular}

Source: Ndiaye, Lab'Urba, 2011

Nous avons choisi ces groupes de variables en prenant en compte les incidences qu'ils pourraient avoir sur les niveaux et conditions de vie des ménages. Le système d'assainissement à Dakar est fonction du type de quartier. Le réseau d'égout et la collecte porte à porte des ordures ménagères n'existent généralement que dans les quartiers lotis et dans certaines zones proches du centre-ville. Les zones non loties ne sont pas dotées de réseau d'assainissement et, du fait de l'impraticabilité des voies de desserte, les véhicules de collecte des ordures ne peuvent y accéder.

15 Nous pouvons citer, entre autres, la population, les activités, l'équipement, l'assainissement, l'ethnie, la confrérie, l'habitat, l'instruction, etc.

16 Ces variables nous semblent pertinentes pour effectuer une classification des districts de recensement (DR). L'objectif final était d'aboutir à l'identification des districts qui avaient les mêmes caractéristiques par rapport aux variables retenues. 
Le type de logement et le niveau d'équipement nous renseignent globalement sur le niveau de vie et de revenu des ménages. En vertu de la réglementation urbaine, les constructions sont généralement en dur, dans les zones loties, structurées, alors que dans les zones non structurées, elles sont faites avec des matériaux précaires. La disponibilité de matériel électroménager nous renseigne aussi sur le niveau de revenu des ménages. Le niveau d'instruction et la pratique d'une activité professionnelle ou scolaire déterminent l'insertion socioprofessionnelle. Il apparaît que les différentes variables retenues pourraient avoir des incidences sur les niveaux et conditions de vie des populations et détermineraient des formes et des types spécifiques d'occupation de l'espace. Nous partons de l'hypothèse que ces variables pourraient permettre de discrétiser notre espace en sous-ensembles homogènes, présentant des caractéristiques distinctifs.

\section{Choix de méthodes d'analyse multivariées}

Notre démarche méthodologique, plus connue sous le concept d'écologie factorielle, nous permet d'étudier les divisions sociales et spatiales dans les quartiers. Cette approche, qui permet de révéler la mosaïque sociorésidentielle à des niveaux très détaillés, intègre des éléments de la sociologie urbaine, de la géographie et des statistiques.

Pour faire notre travail de typologie de l'espace urbain, nous utilisons deux méthodes d'analyse multidimensionnelle: l'analyse en composante principale (ACP) et la classification ascendante hiérarchique $(\mathrm{CAH})$. L'ACP permet, à partir d'un tableau rectangulaire de données comportant les valeurs de $p$ variables quantitatives ( 28 variables regroupées en 5 groupes) pour $n$ unités (appelées aussi individus, qui représentent les districts de recensement), de faire des représentations géométriques de ces unités et de ces variables. Les représentations des unités permettent de voir s'il existe une structure, non connue a priori, sur cet ensemble d'unités. L'ACP permet aussi de traiter un nombre important de variables sans établir de choix a priori des variables discriminantes (Duby et Robin, 2006).

Les méthodes quantitatives et qualitatives permettent d'objectiver les faits sociaux. L'ACP est une méthode descriptive permettant d'analyser un tableau de données quantitatives (Busca et Toutain, 2009). Ces outils mathématiques ont pris naissance au début du XXe siècle chez les psychologues américains et anglais, pour ensuite se développer dans d'autres domaines. En géographie, l'introduction de cette méthode a permis une interprétation plus fine de la mosaïque sociale qui structure les espaces urbains. Appelée «écologie factorielle» lorsqu'elle est appliquée à l'étude spatiale de variables tirées du recensement, «cette méthode d'analyse permet le regroupement des variables qui possèdent des caractéristiques communes» (Viaud, 2006: 303). Le terme même «d'écologie urbaine factorielle est apparu pour la première fois en 1965 sous la plume de Frank L. Sweetser (1965)» (Madoré, 2005 : 45).

Les travaux de l'école de Chicago ont permis de structurer l'espace urbain autour du statut socioéconomique, familial et ethnique des habitants. Ce modèle d'organisation de la ville en zones concentriques a été décrit par Ernest W. Burgess à partir du cas de Chicago (1924). L'économiste Homer Hoyt (1939) a proposé aussi un modèle en secteur, qui introduit le rôle des axes de transport, permettant une rente de situation dans l'utilisation du sol. Les géographes Harris et Ullman (1945) ont aussi proposé le modèle de la ville articulée autour de noyaux multiples. Ce modèle insiste sur la 
dimension polynucléaire ou polycentrique de la ville dont la structure repose encore sur les réseaux de transports (Lajoie, 2007). Les contradictions qui existaient entre ces trois modèles ont été résolues avec l'avènement des techniques quantitatives et de l'écologie factorielle, à l'initiative des géographes et des sociologues nordaméricains (Madoré, 2005).

La méthode de l'ACP a fait l'objet de critique de la part de certains chercheurs, qui considèrent que cette «approche, après avoir révélé les grandes lignes de l'organisation de l'espace résidentiel, s'empêtrait dans une sorte de "spatialisme" occultant les dynamiques sociales productrices de cette organisation» (Villeneuve et al., 2009). C'est pour éviter de tomber dans ce travers que nous avons décidé de collecter des données à l'échelle des différentes zones à identifier, pour rendre compte de cette dynamique sociale. Cependant, cette méthode est toujours considérée comme «l'approche multivariée la plus versatile [sic] et la mieux apte à rendre compte de toute la complexité de la structure des aires sociales» (Viaud, 2006: 303).

Considérant la nature des variables, quantitatives continues, notre choix s'est porté sur les ACP et la CAH. L'ACP a été faite pour chacun des cinq groupes de variables avec le logiciel Statistica. Vu le nombre important de variables, l'ACP devra nous permettre d'avoir une information synthétique. Notre objectif était de déterminer, pour chaque groupe de variables, les axes factoriels les plus significatifs. Les composantes principales ou axes factoriels «sont construits de façon à réduire au maximum les distances entre les individus selon le critère des moindres carrés. [...] Chaque axe synthétise, à partir de plusieurs variables, un "optimum de données" permettant de positionner sur un plan orthonormé les individus en fonction de leur niveau de ressemblance ou de dissemblance» (Busca et Toutain, 2009: 21). Dans le cadre de cette recherche, nous avons eu recours au critère de Kaiser, qui privilégie, dans une ACP normée, les axes dont la valeur propre est supérieure à 1. Cette méthode nous a permis de retenir 10 axes factoriels ( 2 axes par groupe de variables) sur lesquels nous allons appliquer une classification ascendante hiérarchique (CAH). Selon Madoré (2005 : 54), «la classification ascendante hiérarchique apparaît comme une méthode rigoureuse de taxinomie, qui est particulièrement bien adaptée pour l'étude de la configuration socio-spatiale d'une agglomération».

Le travail de classification a été effectué avec le logiciel Philcarto ${ }^{17}$. La classification permet de distinguer les groupes d'individus (ici nos districts de recensement) ayant un comportement homogène. En effet, «la méthode de classification consiste à grouper les individus en classes. Les individus dont les caractéristiques sont proches vont se retrouver dans la même classe et ceux dont les caractéristiques sont différentes vont se retrouver dans des classes différentes» (Minvielle et Souiah, 2003: 73). Avec le logiciel, nous avons procédé à une classification ascendante hiérarchique permettant de discrétiser les districts de recensement suivant les 10 axes factoriels retenus.

17 Philcarto est un logiciel de cartographie des données statistiques, largement utilisé en sciences sociales. Deux autres logiciels, Phildigit et ShapeSelect assurent la numérisation des fonds de carte «[...]. Le développement de ce trio de logiciels, qui s’est déroulé sur une quinzaine d'années, a [...] permis d'aboutir à un produit stable, didactique et correct sur le plan scientifique» (Waniez, 2010). 


\section{Un espace urbain hétérogène et complexe}

Le travail nous a permis d'avoir comme extrant une carte de typologie (figure 4) montrant toute l'hétérogénéité de notre espace d'étude. Les districts de recensement ayant les mêmes caractéristiques par rapport aux axes factoriels seront de la même teinte.

\section{Figure 4 Typologie de la zone urbaine de Dakar en quatre classes}

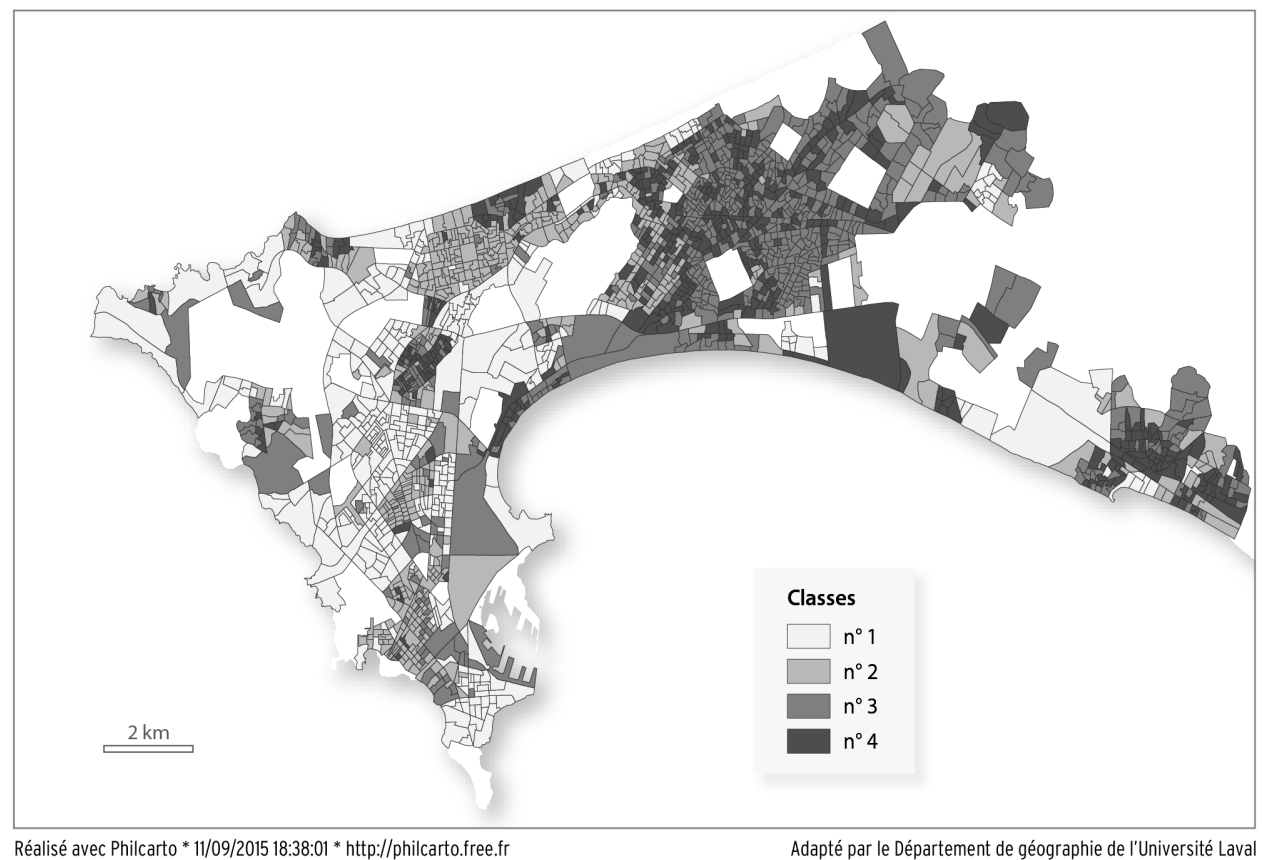

Quatre classes, définissant autant de types de DR, sont sorties de nos analyses:

- classe 1 avec 391 DR: elle est présente principalement dans le département de Dakar et recoupe les quartiers résidentiels (Plateau, Mermoz, SICAP, etc.) ;

- classe 2 avec 511 DR: elle correspond aux quartiers d'habitat régulier de type populaire (Médina, Grand-Dakar, Parcelles assainies, etc.) ;

- classe 3 avec 647 DR: elle correspond à l'habitat irrégulier et est majoritairement présente dans les départements de Pikine, Guédiawaye et Rufisque;

- classe 4 avec 447 DR: plus spécifique aux zones de type traditionnel, cette classe est présente dans les zones de Ngor, Ouakam, Yoff, Grand-Yoff, et Hann.

Le résultat obtenu est une typologie de la région de Dakar en quatre classes. Cette typologie permet de faire ressortir toute l'hétérogénéité caractéristique de Dakar, avec des zones structurées, planifiées, à côté de zones irrégulières non planifiées.

Les quatre zones traduisent une certaine différenciation sociospatiale de la région de Dakar. Pour bien appréhender les caractéristiques des différentes zones, nous avons 
choisi de réaliser des enquêtes à l'échelle des ménages. Lors de ces enquêtes, les zones regroupant les industries et les équipements ont été exclues de nos analyses, du fait de leur faible densité démographique et de leur vocation non résidentielle.

\section{Retour sur le terrain: production de matériaux empiriques}

Ce travail de terrain s'inscrit dans une approche comparative, pour voir s'il existe des divergences ou des similitudes par rapport aux caractéristiques sociospatiales et sociodémographiques des ménages. Les enquêtes de terrain se sont déroulées en deux phases: la première, en avril 2011, et la deuxième, en juin. Les enquêtes ont été réalisées avec l'appui des étudiants de niveau licence en gestion urbaine de l'École Supérieure d'Économie Appliquée. La formation des enquêteurs (au nombre de 18), pour leur permettre de s'approprier l'outil de collecte, a été effectuée sur deux jours. Une enquête test a été réalisée pour corriger les dysfonctionnements et les mauvaises compréhensions de certaines questions. Les interviews ont été faites en face à face, ce qui a permis un taux de réponse élevé et une qualité des données, grâce à la présence et au contrôle de l'enquêteur. Les enquêtes ont été réalisées durant les jours ouvrables (du lundi au vendredi). Lorsque les personnes étaient absentes, un questionnaire leur était laissé, et les enquêteurs retournaient les voir le samedi ou le dimanche suivant pour les rencontrer et éventuellement les aider à remplir le formulaire.

La taille de notre échantillon, 450 ménages, se justifie par notre objectif de disposer de données représentatives (tableau 2) ${ }^{18}$. Cet échantillon a été réparti au prorata du nombre de ménages à rencontrer dans chaque commune d'arrondissement, tout en respectant la classification effectuée précédemment. Il faut préciser qu'à l'échelle des communes d'arrondissement, le choix des ménages a été fait au niveau des districts de recensement.

\section{Tableau 2 Répartition du nombre de ménages et d'individus interviewés selon les départements}

\begin{tabular}{ccc}
\hline Département & Nombre de ménages & Nombre d'individus \\
\hline Dakar & 210 & 649 \\
Pikine & 138 & 405 \\
Guédiawaye & 57 & 170 \\
Rufisque & 31 & 103 \\
Total & $\mathbf{4 3 6}$ & $\mathbf{1 3 2 7}$ \\
\hline
\end{tabular}

Le questionnaire utilisé est scindé en deux parties: une partie ménage et une partie individu. La partie ménage contient les questions concernant les caractéristiques sociodémographiques des membres du ménage, les caractéristiques du logement, les équipements de transport du ménage et l'accès aux services sociaux de base. Elle est administrée à une seule personne de référence, notamment le chef de ménage ou son épouse. La deuxième partie concerne les individus du ménage âgés de plus de sept ans. Chaque individu est interrogé sur ses activités, professionnelles ou scolaires, son insertion sociale et ses opinions sur les modes de transport ${ }^{19}$.

18 En annexe 1, la répartition des individus interviewés par commune est présentée plus en détails.

19 Il faut préciser que toutes les variables du questionnaire utilisé dans le cadre de notre travail de thèse de doctorat n’ont pas été incluses dans cette recherche. 
Le choix des ménages à interviewer dans chaque DR s'est effectué d'une manière aléatoire, à partir du principe que les DR sont homogènes. À l'issue du dépouillement des questionnaires, nous nous sommes retrouvés avec un nombre de 436 questionnaires ménage valides; 14 ont été éliminés parce que mal complétés. Au total, nous avons eu un échantillon de 1327 individus interrogés.

Les données de terrain doivent nous permettre de mieux mettre en exergue la différenciation sociospatiale à travers l'estimation de certains indicateurs pertinents. Il s'agit donc de montrer que ces espaces, bien que proches, présentent des caractéristiques spécifiques.

\section{Une différenciation sociospatiale très marquée}

La synthèse des données issues des enquêtes de terrain nous a permis de mettre en exergue les spécificités de chacune des zones. Cinq groupes d'indicateurs sont retenus pour analyser les caractéristiques des différentes zones: l'habitat, l'accès aux services sociaux de base, le niveau de revenu, l'équipement des ménages et l'activité pratiquée (tableau 3).

\section{Tableau 3 Synthèse des caractéristiques des différentes zones}

\begin{tabular}{|c|c|c|c|c|}
\hline Indicateurs & $\begin{array}{c}\text { Zone } \\
\text { irrégulière }\end{array}$ & $\begin{array}{c}\text { Zone } \\
\text { populaire }\end{array}$ & $\begin{array}{c}\text { Zone } \\
\text { résidentielle }\end{array}$ & $\begin{array}{c}\text { Zone } \\
\text { traditionnelle }\end{array}$ \\
\hline Nombre de personne par pièce & 1,13 & 1,28 & 0,48 & 2,52 \\
\hline$\%$ de zone non aménagée & $34,3 \%$ & $9 \%$ & $8,6 \%$ & $32 \%$ \\
\hline Fréquence des inondations & $64,9 \%$ & $23,3 \%$ & $14,4 \%$ & $18,8 \%$ \\
\hline Prix du $\mathrm{m}^{2}$ de terrain & 50977 & 177088 & 184065 & 97544 \\
\hline $\begin{array}{l}\% \text { de ménages à moins de } 20 \text { min d'une école } \\
\text { primaire }\end{array}$ & $78,4 \%$ & $68,4 \%$ & $85,2 \%$ & $76,2 \%$ \\
\hline \% de ménages à moins de 20 min d'un lycée & $35 \%$ & $36,4 \%$ & $52,9 \%$ & $80 \%$ \\
\hline $\begin{array}{l}\text { \% de ménages à moins de } 20 \text { min d'un centre } \\
\text { de santé }\end{array}$ & $66 \%$ & $81,1 \%$ & $84 \%$ & $69,6 \%$ \\
\hline \% de ménages à moins de 20 min d'un hôpital & $31,3 \%$ & $40,9 \%$ & $37,5 \%$ & $27,3 \%$ \\
\hline \% de ménages à moins de 20 min d'un marché & $53,3 \%$ & $82,7 \%$ & $66,7 \%$ & $64,7 \%$ \\
\hline $\begin{array}{l}\% \text { de ménages situé à moins de } 10 \text { min d'un } \\
\text { arrêt }\end{array}$ & $81,6 \%$ & $83,3 \%$ & $84,1 \%$ & $89,2 \%$ \\
\hline $\begin{array}{l}\text { \% de population ayant un salaire supérieur à } \\
300000 \text { francs CFA }\end{array}$ & $2 \%$ & $6,4 \%$ & $15 \%$ & $4,4 \%$ \\
\hline $\begin{array}{l}\% \text { de population ayant un revenu secondaire } \\
\text { supérieur à } 300000 \text { francs CFA }\end{array}$ & $0 \%$ & $2,6 \%$ & $15,6 \%$ & $0 \%$ \\
\hline \% de ménages équipés d'un véhicule tout genre & $17 \%$ & $27,8 \%$ & $52,2 \%$ & $45,2 \%$ \\
\hline $\begin{array}{l}\text { \% de ménages équipés d'un véhicule } \\
\text { particulier }\end{array}$ & $7,8 \%$ & $17,3 \%$ & $34 \%$ & $22 \%$ \\
\hline$\%$ d'individus ayant un permis de conduire & $17,1 \%$ & $17,5 \%$ & $24 \%$ & $14,6 \%$ \\
\hline$\%$ de population sans instruction & $5,4 \%$ & $3,7 \%$ & $0,8 \%$ & $3,2 \%$ \\
\hline$\%$ d'actifs cadres & $6,5 \%$ & $12,4 \%$ & $16,9 \%$ & $4,8 \%$ \\
\hline \% d'actifs commerçants & $51,6 \%$ & $37,9 \%$ & $18,6 \%$ & $28,6 \%$ \\
\hline
\end{tabular}

Source: Ndiaye, Lab'Urba, 2011 
L'occupation de la zone du Plateau a déclenché le processus d'urbanisation de la région de Dakar. Cette urbanisation s'est étalée à la zone contiguë de la Médina, puis aux autres quartiers construits par les sociétés immobilières (Société immobilière du Cap-Vert et Société nationale des habitats à loyers modérés). La zone résidentielle est constituée principalement des quartiers planifiés. L’analyse de notre tableau montre que la zone résidentielle est située dans des espaces bien aménagés, avec des maisons de grande superficie, caractérisées par l'absence de promiscuité dans les chambres. Grâce à l'existence de réseaux d'évacuation des eaux pluviales, cette zone est exceptionnellement inondée. Elle est habitée par les populations ayant les niveaux de revenu les plus élevés. Le prix du foncier, très élevé, constitue un facteur discriminant en faveur des populations aisées. En outre, les ménages, constitués d'individus ayant un niveau d'instruction très élevé, ont un accès facile aux services sociaux de base. Cette aisance des ménages transparaît aussi au niveau de la motorisation: cette zone a le taux d'équipement en véhicule tout genre et en véhicule particulier le plus élevé. Le taux de possession d'un permis de conduire y est également plus élevé que dans les autres zones. Enfin, cette zone concentre la plus forte proportion de cadres et la plus faible proportion d'actifs dans le secteur commercial. Nous pouvons considérer que c'est la zone des populations nanties, ayant un niveau de vie et de revenu très élevé.

Les zones populaires proches de la zone résidentielle sont occupées par la classe moyenne. La Médina et les Parcelles assainies sont leurs principaux quartiers. Le premier a été construit durant la colonisation pour recevoir les populations indigènes «chassées » du Plateau, et le deuxième, constitué par un grand lotissement sommairement aménagé, a reçu les populations à faible revenu qui n’avaient pas la possibilité d'accéder financièrement aux logements produits par les sociétés immobilières. Leur proximité du centre-ville et des équipements structurants fait que ces quartiers ont connu un changement de catégorie sociale. Avec le renchérissement du prix du foncier et de l'immobilier, beaucoup de propriétaires ont préféré vendre leur propriété pour aller acheter une maison plus loin, dans les zones moins bien desservies. Ce secteur populaire est caractérisé par une très forte concentration démographique et une promiscuité dans les habitations. Celles-ci sont aménagées sommairement et font l'objet d'inondations. Du fait de la proximité de la zone centre, la terre y coûte très cher. Cette proximité fait aussi que les populations ont un accès relativement facile aux services urbains. Le taux de possession d'un véhicule y est assez faible, bien qu'on y trouve une proportion relativement élevée de cadres. Une part assez importante de la population du secteur a un permis de conduire et on y dénombre aussi une proportion assez élevée de résidants dans le secteur du commerce. Ces zones renferment la classe moyenne de la ville de Dakar, constituée d'actifs dans les secteurs publics et privés formels.

Zones de relocalisation des populations déguerpies durant la colonisation, les quartiers irréguliers concentrent les catégories de population les plus pauvres de l'agglomération dakaroise. Ces zones sont très peu touchées par les interventions des pouvoirs publics. Elles sont très peu dotées en équipements et services sociaux de base et subissent des inondations chaque année. Ce secteur est le plus faiblement aménagé et se caractérise par une forte promiscuité dans les chambres. La pauvreté y est ambiante et les conditions de vie, très difficiles. Le manque d'aménagement et l'occupation irrégulière, avec l'absence de titres de propriété, y rendent l'accès à la terre et donc la propriété foncière relativement faciles. Cette facilité attire les populations pauvres. L'accès aux services urbains est cependant très difficile à cause 
de l'insuffisance de la desserte en transport. Le taux de motorisation des ménages y est très faible. Le secteur est caractérisé aussi par une faible proportion d'employés cadres. La population active est très présente dans le secteur marchand informel, qui demande peu de qualification. Ces quartiers regroupent les populations les plus pauvres de la région de Dakar, et les conditions d'existence y sont très difficiles.

Enfin, les zones traditionnelles constituent les espaces les plus anciennement occupés à Dakar. Bien avant la colonisation, les villages traditionnels étaient habités par les populations léboues. Ces zones non aménagées sont généralement situées au niveau du littoral, une localisation liée à la pratique de la pêche, qui était la principale occupation des populations autochtones. Ces zones traditionnelles sont caractérisées par une promiscuité très forte, qui est source de maladies diverses. Le manque d'aménagement fait qu'elles sont sujettes à des inondations, dont l'ampleur est amoindrie par la proximité de la mer. La proximité des rivages en fait des zones très convoitées, ce qui - la spéculation aidant - augmente les prix du foncier et de l'immobilier. L'accès aux services urbains est relativement facile. Cependant, la proximité de la mer, avec un climat très agréable, amène un début d' «accaparement» de ces espaces par les classes aisées. Le taux de possession d'un véhicule particulier y est relativement élevé par rapport aux autres zones présentant les mêmes caractéristiques. La proportion de cadres et de commerçants est relativement faible. Nous notons cependant une importante proportion de personnes actives dans le secteur halieutique (la pêche est l'activité traditionnelle des Lébous). Cette partie de Dakar présente les caractéristiques d'une zone mixte: du fait de son attrait, lié à la proximité de la mer et à l'accessibilité des prix du foncier, on constate de plus en plus la présence d'une populations aisée, ce qui n'empêche pas des populations relativement pauvres d'y habiter.

\section{Conclusion}

Il ressort de nos analyses que l'agglomération dakaroise est sujette à un étalement urbain consécutif à l'échec des politiques de planification du développement et à la déréglementation du marché du foncier et de l'immobilier, qui ont été sources d'exclusion des populations à faible revenu. L'analyse de ce territoire étalé montre un espace complexe, animé d'une dynamique de différenciation sociospatiale très marquée. Les résultats de cette recherche s'inscrivent bien dans ceux obtenus lors des travaux successifs menés sur Dakar (Seck, 1970; Vernière, 1973 et 1977; Lessault et Sakho, 2008 ; Lessault et Imbert, 2013) et sur d'autres espaces des pays du Sud (Bertrand, 2011).

L'une des difficultés majeures pour les chercheurs, plus encore pour ceux des pays du Sud, est de disposer de données spatialisées de bonne qualité. Ainsi, pour s'extraire des études sectorielles, s'intéressant à des espaces confinés, il est nécessaire de recourir à des démarches méthodologiques permettant d'analyser des espaces plus étendus et complexes. Cependant, la disponibilité d'informations sur le temps long, notamment les données des recensements généraux de la population de 1976 et de 1988, aurait pu nous permettre de rendre nos analyses sur l'évolution des configurations sociospatiales plus dynamiques et de mieux intégrer la question des temporalités.

Nos analyses montrent aussi que les écarts entre zones ne sont pas aussi marqués pour certains indicateurs. En effet, leur évaluation montre que certaines zones ne sont pas homogènes et que les ratios obtenus cachent des disparités internes. 
Le centre-ville et ses environs attirent parce que concentrant les fonctions urbaines les plus importantes (administrative, commerciale, économique, etc.). Cependant, une analyse beaucoup plus fine et désagrégée permet de mettre en évidence une tendance à l'émergence de nouvelles centralités (Ndiaye, 2011). Ainsi, donc, l'attrait du centre-ville est à relativiser selon le type de mobilité observé: le centre est attrayant pour certains déplacements, mais les mobilités internes aux quartiers de Dakar, y compris périphériques, restent dominantes. L’observation de ces mobilités urbaines peut révéler d'autres formes ou permettre une autre lecture des différenciations sociospatiales à Dakar.

L'étalement de la ville de Dakar, conjugué à l'apparition de ces nouvelles centralités ainsi que la mise en œuvre de projets d'infrastructures structurantes (autoroute à péage) risqueraient de déclencher de nouveaux processus succeptibles de reconfigurer les dynamiques de différenciation sociospatiales.

\section{Remerciements}

Nous remercions la Fondation Mc Namara pour son appui dans le cadre de la réalisation de ce travail de recherche. 


\section{Annexe 1 Répartition du nombre d'individus interviewés selon les communes}

\begin{tabular}{lc|lc}
\hline Commune d'arrondissement & $\begin{array}{c}\text { Nombre } \\
\text { d'interviewés }\end{array}$ & Commune d'arrondissement & $\begin{array}{c}\text { Nombre } \\
\text { d'interviewés }\end{array}$ \\
\hline Liberté & 25 & Yeumbeul Nord & 30 \\
Grand Yoff & 65 & Djida Thiaroye Kao & 33 \\
Grand Dakar & 39 & Guinaw Rail Nord & 31 \\
HLM & 16 & Thiaroysurmer & 19 \\
Mermoz & 28 & Tivaouane Diak Sao & 4 \\
Biscuiterie & 48 & Yeumbeul Sud & 16 \\
Cambérène & 28 & Keur Massar & 15 \\
Dieuppeul & 29 & Pikine Est & 15 \\
Fann-Point E & 14 & Pikine Nord & 38 \\
Gueule Tapée & 68 & Thiaroye Gare & 25 \\
Hann Bel-Air & 21 & Mbao & 27 \\
Médina & 74 & Diamagueune & 74 \\
Ngor & 12 & Guinaw Rail Sud & 23 \\
Ouakam & 27 & Golf & 45 \\
Parcelles assainies & 102 & Médina Gounass & 20 \\
Patte d'Oie & 18 & Ndiarem Limamoulaye & 28 \\
Plateau & 10 & Sam Notaire & 23 \\
Yoff & 25 & Wakhinan & $\mathbf{1 3 2 7}$ \\
Dalifort & 20 & Rufisque Est & 54 \\
Malika & 15 & Rufisque Nord & 38 \\
Pikine Ouest & 20 & Rufisque Ouest & 46 \\
\hline & & & 19 \\
\hline & & & \\
\hline
\end{tabular}




\section{Bibliographie}

ANSD (AGENCE NATIONALE DE LA STATISTIQUE ET DE LA DÉMOGRAPHIE) (2005) Enquête sénégalaise auprès des ménages. Dakar.

ASCHER, François (2004) Le sens du mouvement: modernités et mobilités. Dans Sylvain Allemand, François Ascher et Jacques Lévy (dir.) Le sens du mouvement. Institut ville en mouvement, Paris, Belin.

BERTRAND, Monique (2011) De Bamako à Accra, mobilités urbaines et ancrages locaux en Afrique de l'Ouest. Paris, Éditions Karthala.

BUSCA, Didier et TOUTAIN, Stéphanie (2009) Analyse factorielle simple en sociologie. Méthodes d'interprétation et études de cas. Bruxelles, De Boeck Supérieur.

DIAZ, Olvera Lourdes et GODARD, Xavier (2002) P comme pauvreté ou le rôle du transport pour la combattre. Dans Xavier Godard (dir.) Les transports et la ville en Afrique au sud du Sahara. Paris, Éditions Kharthala-Inrets, p. 249-262.

DUBY Camille, ROBIN Stéphane (2006) Analyse en composantes principales. Institut National Agronomique Paris Grignon. Work document, 54 p. [En ligne]. http://math.univ-lyon1.fr/ okra/2010Mass41-Algebre/ACP.pdf

FIJALKOW, Yankel (2007) Sociologie de la ville. Paris, Repère.

GRAFMEYER, Yves et AUTHIER, Jean-Yves (2008) Sociologie urbaine. Paris, Armand Colin.

KAUFMANN, Vincent, SAGER, Fritz, FERRARI, Yves et JOYE, Dominique (2003) Coordonner transports et urbanisme. Lausanne, Presses Polytechniques et Universitaires Romandes, 217 p.

LAJOIE, Gilles (2007) Recherches en modélisation urbaine. Université de la Réunion. Mémoire d'habilitation à diriger des recherches.
LESSAULT, David, BEAUCHEMIN, Cris et SAKHO, Papa (2011) Migration internationale et conditions d'habitat des ménages à Dakar. Population vol.66, p. 197-228 [En ligne]. www.cairn.info/ revue-population-2011-1-page-197.htm

LESSAULT, David et IMBERT, Christophe (2013) Mobilité résidentielle et dynamique récente du peuplement urbain à Dakar (Sénégal). Cybergeo: European Journal of Geography [En ligne]. http://cybergeo. revues.org/26146

LESSAULT, David et SAKHO, Papa (2008) Étalement urbain et risques de fragmentation à Dakar. Dans Jean-Marc Zaninetti et Isabelle Maret (dir.) Étalement urbain et villes fragmentées à travers le monde, des théories aux faits. Orléans, Presses Universitaires d'Orléans, p. 95-118.

LOMBARD, Jérôme, BRUEZ, Frank et DIAKHO, Arouna (2006) Les transports sénégalais à l'aune du système-monde. Dans Jérôme Lombard et Evelyne Mesclier (dir.) La mondialisation côté Sud: acteurs et territoires. Paris, Institut de recherche pour le développement, p. 179-202.

MADORÉ, François (2005) La ségrégation sociale dans les villes françaises : réflexion épistémologique et méthodologique. Cahiers de géographie du Québec, vol.49, no 136, p. 45-60 [En ligne]. http://id.erudit. org/iderudit/012108ar

MASSOT, Marie-Hélène et ORFEUIL, Jean-Pierre (2004) Les mobilités urbaines dans 20 ans. Dans Sylvain Allemand, François Ascher et Jacques Lévy (dir.) Le sens du mouvement. Institut ville en mouvement, Paris, Belin, p. 119-128.

MBOW, Lat Soukabé (1992) Dakar: croissance et mobilité urbaine. Université Paris X Nanterre, thèse de doctorat d'État.

MBOW, Lat Soukabé (1992) Les politiques urbaines : gestion et aménagement. Dans Momar-Coumba Diop (dir.) Sénégal. Trajectoires d'un État Dakar. Dakar, Sénégal, Codesria, p. 205-231. 
MINVIELLE, Erwann et SOUIAH, Sid'Ahmed (2003) Analyse statistique et spatiale. Nantes, Édition du temps.1

NDIAYE, Ibrahima (2009) Mutations socio-économiques et spatiales dans les espaces urbains à Dakar: cas de la Médina. Communication au colloque Pôle Ville, Université Paris Est.

NDIAYE, Ibrahima (2011) Déplacements quotidiens et nouvelles centralités à Dakar, Sénégal. Revue de géographie du LARDYMES/Université de Lomé, n 7 , p. 141-151.

PÉTUAUD-LÉTANG, Michel (2010) Comment la ville prend-elle forme? Dans Jean-Pierre Augustin et Michel Favory (dir.) 50 questions à la ville: comment penser et agir sur la ville. Pessac, Maison des Sciences de l'Homme d'Aquitaine.

POUYANNE, Guillaume (2004) Formes urbaines et mobilité quotidienne. Université Montesquieu Bordeaux IV, thèse de doctorat ès sciences économiques.

SAKHO, Papa (1985) Problématique de la rénovation urbaine d'un quartier de Dakar: cas des sous-secteurs de la Médina Est. Université Cheikh Anta Diop de Dakar - Sénégal, département de géographie, mémoire de maîtrise.

SALEM, Gérard (1998) La santé dans la ville. Géographie d'un petit espace dense: Pikine (Sénégal). Paris, Éditions Karthala et Office de la recherche scientifique et technique outre-mer (ORSTOM).

SECK, Assane (1970) Dakar, métropole ouestafricaine. Dakar, Institut fondamental d'Afrique noire (IFAN).

SINOU, Alain (1985) Idéologies et pratiques de l'urbanisme dans le Sénégal colonial. Paris, École des hautes études en sciences sociales (EHESS), thèse de $3^{\text {e }}$ cycle.
SY, Mamadou (1991) Le droit foncier intermédiaire et la croissance urbaine dans les pays d'Afrique de l'ouest: le cas de Pikine irrégulier. Dans Émile Le Bris, Annik Osmont, Ardjouma Ouattara, Fatoumata Kinda, Mansorh Sy, Catherine Goislard et Alphonse Yapi Diahou (dir.) Contribution à la connaissance du droit foncier intermédiaire dans les villes d'Afrique de l'Ouest. Paris, Office de la recherche scientifique et technique outre-mer (ORSTOM), p. 106-128.

TALL, Serigne Mansour (2009) Investir dans la ville africaine - Les émigrés et l'habitat à Dakar. Paris, Éditions Karthala.

VERNIÈRE, Marc (1973) Pikine, ville nouvelle de Dakar, un cas de pseudo-urbanisation. Espace géographique, n²2, p. 107-126.

VERNIÈRE, Marc (1977) Les oubliés de l'«haussmannisation» dakaroise: crise du logement populaire et exploitation rationnelle des locataires. Espace géographique, $\mathrm{n}^{0} 1$, p. 5-23.

VIAUD, Gilles (2006) Deux chantiers de l'écologie factorielle: le genre et les petites villes. Cahiers de géographie du Québec, vol. 50, no 141, p. 303-309 [En ligne]. http://id.erudit.org/iderudit/014870ar

VILLENEUVE, Paul, PELLETIER, Mathieu et THÉRIAULT, Marius (2009) La dynamique sociale des quartiers urbains: essai méthodologique. Espace populations sociétés, $\mathrm{n}^{\circ} 1$ [En ligne]. http://eps.revues. org/index3617.html

WANIEZ, Philippe (2010) Philcarto: histoire de vie d'un logiciel de cartographie. Cybergeo: European Journal of Geography [En ligne]. http://cybergeo.revues. org/23076

WIEL, Marc (2010) Étalement urbain et mobilités. Paris, Programme de recherche et d'innovation dans les transports terrestres (PREDIT), La Documentation française. 